\title{
Multi-Agent Task-Oriented Dialog Policy Learning with Role-Aware Reward Decomposition
}

\author{
Ryuichi Takanobu, Runze Liang, Minlie Huang* \\ Institute for AI, BNRist, DCST, Tsinghua University, Beijing, China \\ \{gxly19, liangrz15\}@mails.tsinghua.edu.cn, aihuangetsinghua.edu.cn
}

\begin{abstract}
Many studies have applied reinforcement learning to train a dialog policy and show great promise these years. One common approach is to employ a user simulator to obtain a large number of simulated user experiences for reinforcement learning algorithms. However, modeling a realistic user simulator is challenging. A rule-based simulator requires heavy domain expertise for complex tasks, and a data-driven simulator requires considerable data and it is even unclear how to evaluate a simulator. To avoid explicitly building a user simulator beforehand, we propose Multi-Agent Dialog Policy Learning, which regards both the system and the user as the dialog agents. Two agents interact with each other and are jointly learned simultaneously. The method uses the actorcritic framework to facilitate pretraining and improve scalability. We also propose Hybrid Value Network for the role-aware reward decomposition to integrate role-specific domain knowledge of each agent in task-oriented dialog. Results show that our method can successfully build a system policy and a user policy simultaneously, and two agents can achieve a high task success rate through conversational interaction.
\end{abstract}

\section{Introduction}

Dialog policy, which decides the next action that the dialog agent should take, plays a vital role in a task-oriented dialog system. More recently, dialog policy learning has been widely formulated as a Reinforcement Learning (RL) problem (Su et al., 2016; Peng et al., 2017; He et al., 2018; Zhao et al., 2019; Zhang et al., 2019; Takanobu et al., 2019), which models users as the interactive environment. Since RL requires much interaction for training, it is too time-consuming and costly to interact with real users directly. The most common way is first

\footnotetext{
${ }^{*}$ Corresponding author
}

to develop a dialog agent with a user simulator that mimics human behaviors in an offline scenario.

Designing a reliable user simulator, however, is not trivial and often challenging as it is equivalent to building a good dialog agent. With the growing needs for the dialog system to handle more complex tasks, it will be much challenging and laborious to build a fully rule-based user simulator, which requires heavy domain expertise. Datadriven user simulators have been proposed in recent studies (Kreyssig et al., 2018; Shi et al., 2019), but they require a considerable quantity of manually labeled data, most of which regard the simulator as a stationary environment. Furthermore, there is no standard automatic metric for evaluating these user simulators, as it is unclear to define how closely the simulator resembles real user behaviors.

In this paper, we propose Multi-Agent Dialog Policy Learning (MADPL), where the user is regarded as another dialog agent rather than a user simulator. The conversation between the user and the system is modeled as a cooperative interactive process where the system agent and the user agent are trained simultaneously. Two dialog agents interact with each other and collaborate to achieve the goal so that they require no explicit domain expertise, which helps develop a dialog system without the need of a well-built user simulator. Different from existing methods (Georgila et al., 2014; Papangelis et al., 2019), our approach is based on actor-critic framework (Barto et al., 1983) in order to facilitate pretraining and bootstrap the RL training. Following the paradigm of centralized training with decentralized execution (CTDE) (Bernstein et al., 2002) in multi-agent RL (MARL), the actor selects its action conditioned only on its local stateaction history, while the critic is trained with the actions of all agents.

It should be noted that the roles of two agents are different though they interact with each other 


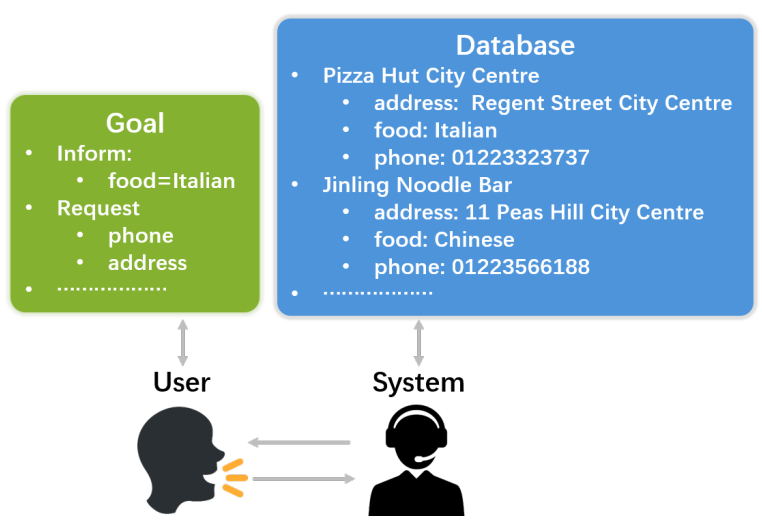

Figure 1: The user has his/her own goal to be accomplished and the system is provided with an interface to access an external database. Both agents can only obtain information from the other side via communication.

in a cooperative setting. As shown in Fig. 1, only the user agent knows the user goal, while only the system agent can access the backend database. The user agent should express the requirements completely in an organized way, and the system should respond with useful information accurately and immediately. So it is inappropriate to apply simple self-play RL (Silver et al., 2017; Lewis et al., 2017) that views two agents as the same agent in this task. To address this issue, the system and the user are viewed as two asymmetric agents in MADPL. We introduce Hybrid Value Network (HVN) for roleaware reward decomposition. It decomposes the reward into two parts: one is the role-specific reward that focuses on its local target, and the other is the global reward that represents the shared goal.

To evaluate the proposed approach, we conduct our experiments on a multi-domain, multiintent task-oriented dialog corpus, MultiWOZ (Budzianowski et al., 2018). The corpus involves high dimensional state and action spaces, multiple decision making in one turn, which makes it more difficult to get a good system policy as well as a good user policy. The experiments demonstrate that MADPL can successfully build a system policy as well as a user policy with the aid of HVN, and two agents can achieve high task success rate in complex tasks by interacting with each other as well as with benchmark policies.

To summarize, our contributions are in three folds:

- We apply actor-critic based multi-agent reinforcement learning to learn the task-oriented dialog policy to facilitate pretraining and avoid explicitly building a user simulator.

- We propose Hybrid Value Network for reward decomposition to deal with the asymmetric role issue between the system agent and the user agent in the task-oriented dialog.

- We conduct in-depth experiments on the multidomain, multi-intent task-oriented dialog corpus to show the effectiveness, reasonableness and scalability of our algorithm.

\section{Related Work}

\subsection{Multi-Agent Reinforcement Learning}

The goal of RL is to discover the optimal strategy $\pi^{*}(a \mid s)$ of the Markov Decision Process, which can be extended into the $N$-agent setting, where each agent has its own set of states $\mathcal{S}_{i}$ and actions $\mathcal{A}_{i}$. In MARL, the state transition $s=\left(s_{1}, \ldots, s_{N}\right) \rightarrow$ $s^{\prime}=\left(s_{1}^{\prime}, \ldots, s_{N}^{\prime}\right)$ depends on the actions taken by all agents $\left(a_{1}, \ldots, a_{N}\right)$ according to each agent's policy $\pi_{i}\left(a_{i} \mid s_{i}\right)$ where $s_{i} \in \mathcal{S}_{i}, a_{i} \in \mathcal{A}_{i}$, and similar to single RL, each agent aims to maximize its local total discounted return $R_{i}=\sum_{t} \gamma^{t} r_{i, t}$.

Since two or more agents learn simultaneously, the agents continuously change as the training proceeds, therefore the environment is no longer stationary. Many MARL algorithms (Lowe et al., 2017; Foerster et al., 2018; Rashid et al., 2018) have been proposed to solve challenging problems. Most of them use the CTDE framework to address the non-stationarity of co-adapting agents. It allows the policies to use extra information to ease training, but the learned policies can only use local information (i.e. their own observations) at execution time.

Several studies have demonstrated that applying MARL delivers promising results in NLP tasks these years. While some methods use identical rewards for all agents (Das et al., 2017; Kottur et al., 2017; Feng et al., 2018), other studies use completely separate rewards (Georgila et al., 2014; Papangelis et al., 2019). MADPL integrates two types of rewards by role-aware reward decomposition to train a better dialog policy in task-oriented dialog.

\subsection{User Modeling in Task-Oriented Dialog}

User modeling is essential for training RL-based dialog models, because a large amount of dialog samples are required for RL policy learning, mak- 
ing it impractical to learn with real users directly from the beginning.

There are three main approaches for user modeling. The first approach is to build a rule-based user simulator. Among these methods, the most popular one is agenda-based simulator (Schatzmann et al., 2007; Shah et al., 2018), which is built on hand-crafted rules with a stack-like agenda based on the user goal. The second approach is to build a user simulator from the dialog data (Keizer et al., 2010; El Asri et al., 2016; Kreyssig et al., 2018). Recently, Gür et al. (2018) uses a variational hierarchical seq2seq framework to encode user goal and system turns, and then generate the user response. Shi et al. (2019) uses two decoders with a copy and attention mechanism to predict a belief span first and then decode user utterance. The third approach is to use model-based policy optimization that incorporates a differentiable model of the world dynamics and assumptions about the interactions between users and systems ( $\mathrm{Su}$ et al., 2018; Zhang et al., 2019), but this approach still requires real users or a user simulator for world model learning.

Instead of employing a user simulator, a few methods jointly learn two agents directly from the corpus. Liu and Lane (2017) models the system and the user by iteratively training two policies. Papangelis et al. (2019) make the first attempt to apply MARL into the task-oriented dialog policy, whose algorithm is based on Q-learning for mixed policies. However, it is not well scalable to complex tasks such as multi-domain dialog. Therefore, MADPL uses the actor-critic framework instead to deal with the large discrete action space in dialog.

\section{Multi-Agent Dialog Policy Learning}

We first formally describe the task, and then present the overview of our proposed model. Specifically, given a user goal $G=(C, R)$ composed of the user constraints $C$ (e.g. a Japanese restaurant in the center of the city) and requests $\mathrm{R}$ (e.g. inquiry for address, phone number of a hotel), and given an external database $\mathrm{DB}$ containing all candidate entities and corresponding information, the user agent and system agent interact with each other in a dialog session to fulfill the user goal. There can be multiple domains in $\mathrm{G}$, and two agents have to accomplish all the subtasks in each domain. Both agents can partially observe the environment, i.e. only the user agent knows $\mathrm{G}$, while only the sys-

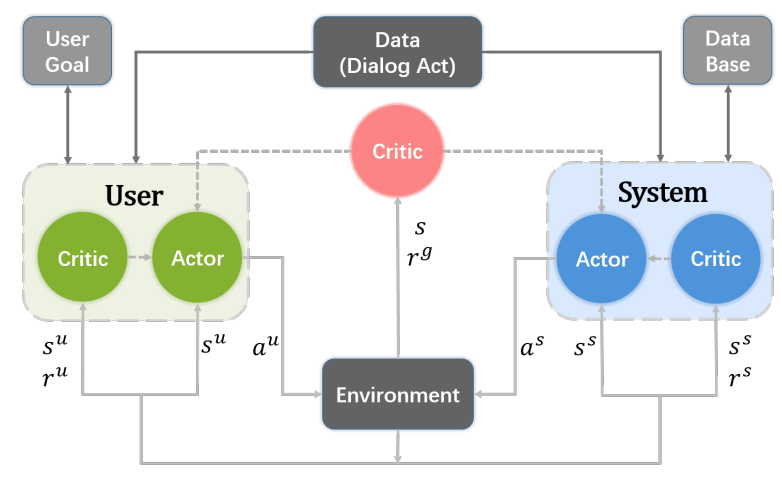

Figure 2: Architecture of MADPL. HVN consists of three critics. Each critic estimates its return based on role-aware reward decomposition, and each actor uses the estimated value to optimize itself.

tem agent can access $\mathrm{DB}$, and the only way to know each other's information is through conversational interaction. Different from ordinary multiagent task setting, two agents in dialog are executed asynchronously. In a single dialog turn, the user agent posts an inquiry first, then the system agent returns a response, and the two communicate alternately. Therefore, each dialog session $\tau$ can be seen as a trajectory of state-action pairs $\left\{\left(s_{0}^{U}, a_{0}^{U}, s_{0}^{S}, a_{0}^{S}\right) ;\left(s_{1}^{U}, a_{1}^{U}, s_{1}^{S}, a_{1}^{S}\right) ; \ldots\right\}$, where the user agent and the system agent make decisions according to each dialog policy $\mu\left(a^{U} \mid s^{U}\right), \pi\left(a^{S} \mid s^{S}\right)$ respectively.

Here we present a novel algorithm, Multi-Agent Dialog Policy Learning (MADPL), as shown in Fig. 2, which can be naturally formulated as a MARL problem. Two agents interact through dialog acts following (Georgila et al., 2014). We choose the actor-critic framework in order to learn an explicitly stochastic dialog policy (actor) for high scalability along with an estimated value function (critic) to bootstrap RL training. Besides, this can facilitate imitation learning to pretrain the dialog policy using human-human dialogs. Since two agents cooperate to reach success, yet their roles are asymmetric in the dialog, we propose Hybrid Value Network (HVN) to decompose the task reward into different parts for better policy learning. Note that our approach is fully data-driven without building a user simulator beforehand, and does not need any other human supervision during training.

In the subsequent subsections, we will first explain the state and action used in two dialog policies. Then we describe how we decompose the reward and the proposed HVN. At last, we present model optimization. 


\subsection{Dialog Policy}

System Policy The system policy $\pi$ decides the system action $a^{S}$ according to the system dialog state $s^{S}$ to give the appropriate response to user agent. Each system action $a^{S}$ is a subset of dialog act set $\mathcal{A}$ as there may be multiple intents in one dialog turn. A dialog act is an abstract representation of an intention (Stolcke et al., 2000), which can be represented in a quadruple composed of domain, intent, slot type and slot value (e.g. [restaurant, inform, food, Italian]). In practice, dialog acts are delexicalized in the dialog policy. We replace the slot value with a count placeholder and refill it with the true value according to the entity selected from the external database DB, which allows the system to operate on unseen values. The system dialog state $s_{t}^{S}$ at dialog turn $t$ is the concatenation of (I) user action at current turn $a_{t}^{U}$; (II) system action at the last turn $a_{t-1}^{U}$; (III) the belief state $b_{t}$ (Williams et al., 2016) that keeps track of constraint slots and request slots supplied by the user agent; and (IV) embedding vectors of the number of query results $q_{t}$ from DB.

User Policy The user policy $\mu$ decides the user action $a^{U}$ according to the user dialog state $s^{U}$ to express its constraint and request to the system agent. Similar to the system policy, the user policy uses delexicalized dialog acts as actions, and the value is refilled according to the user goal G. User dialog state $s_{t}^{U}$ is the concatenation of (I) last system action $a_{t-1}^{S}$; (II) last user action $a_{t-1}^{U}$; (III) the goal state $g_{t}$ that represents the remained constraint and request that need to send; (IV) inconsistency vector $c_{t}$ (Kreyssig et al., 2018) that indicates the inconsistency between the systems response and user constraint $C$. In addition to predicting dialog acts, the user policy outputs terminal signal $T$ at the same time, i.e. $\mu=\mu\left(a^{U}, T \mid s^{U}\right)$.

\subsection{Reward Decomposition}

On the one hand, the roles between the user agent and the system agent are different. The user agent actively initiates a task and may change it during conversation, but the system agent passively responds to the user agent and returns the proper information, so the reward should be considered separately for each agent. On the other hand, two agents communicate and collaborate to accomplish the same task cooperatively, so the reward also involves a global target for both agents. Therefore, we decompose the mixed reward into three parts according to the characteristic of each component. The reward of each part is explained as follows:

System Reward $r_{t}^{S}$ consists of (I) empty dialog act penalty $a_{t}^{S}=\varnothing$; (II) late answer penalty if there is a request slot triggered but the system agent does not reply the information immediately; and (III) task success reward based on the user agent's description.

User Reward $r_{t}^{U}$ consists of (I) empty dialog act penalty $a_{t}^{U}=\varnothing$; (II) early request penalty if the user agent requests for information when there is still a constraint slot remained to inform; and (III) user goal reward whether the user agents have expressed all the constraints $\mathrm{C}$ and requests $\mathrm{R}$.

Global Reward $r_{t}^{G}$ consists of (I) efficiency penalty that a small negative value will be given at each dialog turn; (II) sub-goal completion reward once the subtask of $\mathrm{G}$ in a particular domain is accomplished; and (III) task success reward based on user goal G.

Obviously, each agent should obtain its local reward, and both agents should receive the global reward during the training process. Note that the task success and the user goal reward are only computed at the end of the dialog, and the task success computed in the system reward differs from the one in the global reward.

\subsection{Hybrid Value Network}

The value function aims to estimate the expected return given the current state $V\left(s_{t}\right)=\mathbb{E}\left[R_{t}\right]=$ $\mathbb{E}\left[\sum_{t^{\prime} \geq t} \gamma^{t^{\prime}-t} r_{t^{\prime}}\right]$ so that the policy can directly use the estimated cumulative reward for optimization, without sampling the trajectories to obtain rewards which may cause high variance. Another advantage by applying actor-critic approaches in MARL is that it can integrate with the CTDE framework: the actor of each agent benefits from a critic that is augmented with additional information about the policies of other agents during training. However, a simple centralized critic conditioned on the global state and joint actions cannot well exploit the domain knowledge mentioned above since each part of the overall rewards only depends on a subset of features, e.g. the system reward only depends on the system agent's behaviors.

Inspired by Hybrid Reward Architecture (Van Seijen et al., 2017) that learns a separate Q function, we propose Hybrid Value Network to improve an estimate of the optimal role-aware 
value function. It first encodes the dialog state of each agent to learn a state representation

$$
\begin{aligned}
& h_{s}^{S}=\tanh \left(f_{s}^{S}\left(s^{S}\right)\right), \\
& h_{s}^{U}=\tanh \left(f_{s}^{U}\left(s^{U}\right)\right),
\end{aligned}
$$

where $f(\cdot)$ can be any neural network unit. The value network $V$ is separated into three branches $V^{S}, V^{U}$ and $V^{G}$ for the value of system rewards, user rewards and global rewards, respectively.

$$
\begin{aligned}
V^{S}\left(s^{S}\right) & =f_{S}\left(h_{s}^{S}\right), \\
V^{U}\left(s^{U}\right) & =f_{U}\left(h_{s}^{U}\right), \\
V^{G}(s) & =f_{G}\left(\left[h_{s}^{S} ; h_{s}^{U}\right]\right) .
\end{aligned}
$$

\subsection{Optimization}

The action space for the policies can be very large since we deal with multi-domain, complex dialog tasks, which makes it almost impossible for the RL policies to explore and learn from scratch. So the training process can be split into two stages (Fatemi et al., 2016; Takanobu et al., 2019): pretraining the dialog policy with the conversational corpus first and then using RL to improve the pretrained policies. We use $\beta$-weighted logistic regression for policy pretraining here to alleviate data bias because each agent only generates several dialog acts in one dialog turn

$$
\begin{aligned}
L(X, Y ; \beta)= & -\left[\beta \cdot Y^{T} \log \sigma(X)\right. \\
& \left.+(I-Y)^{T} \log (I-\sigma(X))\right],
\end{aligned}
$$

where $X$ is the state and $Y$ is the action from the corpus in this task.

As for critic optimization, it aims to minimize the squared error between the temporal difference (TD) target $r_{t}+\gamma V\left(s_{t+1}\right)$ and the estimated value $V\left(s_{t}\right)=\mathbb{E}\left[r_{t}+\gamma V\left(s_{t+1}\right)\right]$. Actor-critic algorithms have high variance since the critic is updated too frequently, which has contributed to severe changes in the estimated value, particularly in multi-agent tasks. So we introduce a target network (Mnih et al., 2015) to make the training process more stable. In the context of $\mathrm{HVN}$, it aims to minimize the following loss functions:

$$
\begin{aligned}
L_{V}^{S}(\theta) & =\left(r^{S}+\gamma V_{\theta^{-}}^{S}\left(s^{\prime S}\right)-V_{\theta}^{S}\left(s^{S}\right)\right)^{2}, \\
L_{V}^{U}(\theta) & =\left(r^{U}+\gamma V_{\theta^{-}}^{U}\left(s^{\prime}\right)-V_{\theta}^{U}\left(s^{U}\right)\right)^{2}, \\
L_{V}^{G}(\theta) & =\left(r^{G}+\gamma V_{\theta^{-}}^{G}\left(s^{\prime}\right)-V_{\theta}^{G}(s)\right)^{2}, \\
L_{V} & =L_{V}^{S}+L_{V}^{U}+L_{V}^{G},
\end{aligned}
$$

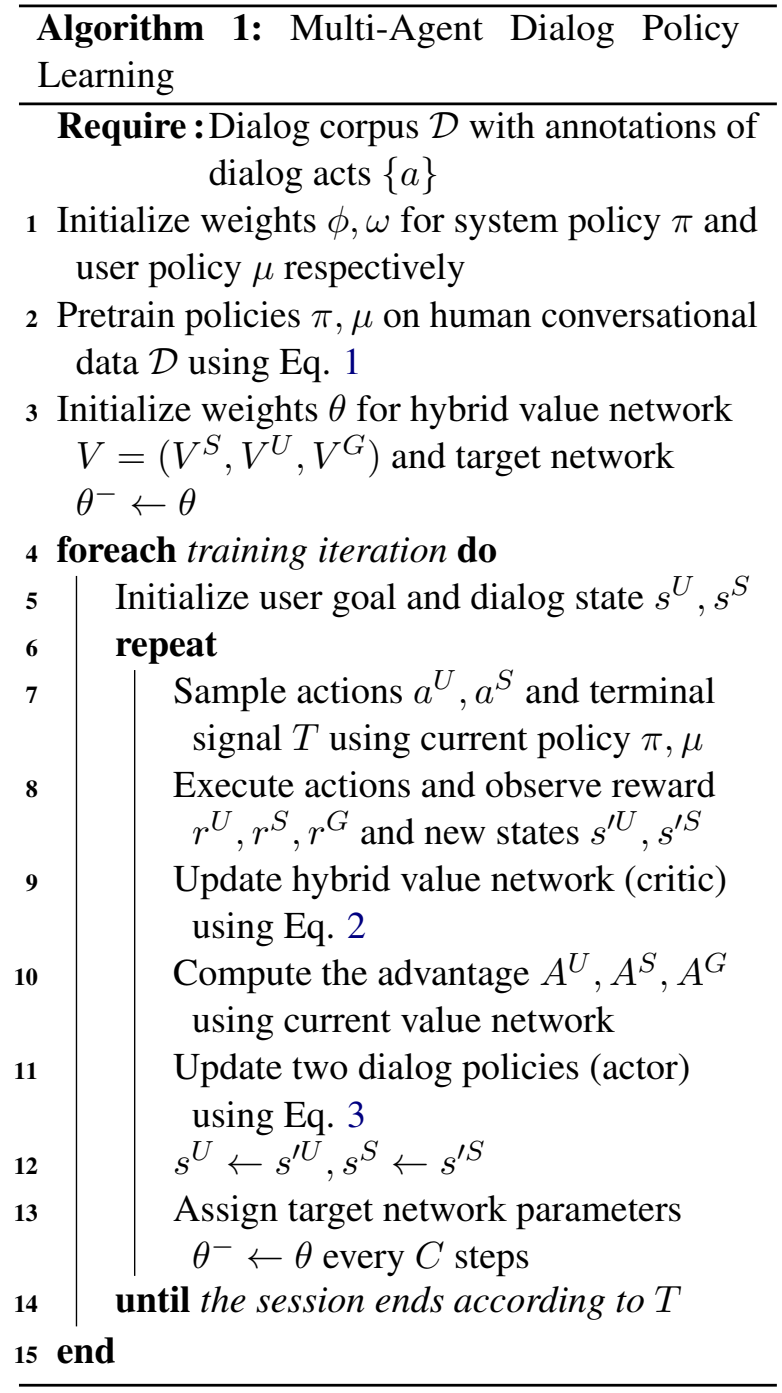

where HVN $V_{\theta}$ is parameterized by $\theta$, and $\theta^{-}$is the weight of target network, and the overall loss $L_{V}$ is the sum of value estimation loss on each component reward.

Each dialog policy aims to maximize all the related returns, e.g. the system policy $\pi$ aims to maximize the cumulative system rewards and global rewards $\mathbb{E}\left[\sum_{t} \gamma^{t}\left(r_{t}^{S}+r_{t}^{G}\right)\right]$. The advantage $A(s)=r+\gamma V\left(s^{\prime}\right)-V(s)$ estimated by the critic can evaluate the new state $s^{\prime}$ and current state $s$ to determine whether the dialog has become better or worse than expected. With the aid of HVN, the sum of the related component advantages can be used to update different agents. By using the loglikelihood ratio trick, the gradients for the system policy and the user policy yield:

$$
\nabla_{\phi} J_{\pi}(\phi)=\nabla_{\phi} \log \pi_{\phi}\left(a^{S} \mid s^{S}\right)\left[A^{S}\left(s^{S}\right)+A^{G}(s)\right],
$$


where the system policy $\pi_{\phi}$ is parameterized by $\phi$ and the user policy $\mu_{\omega}$ by $\omega$.

In summary, a brief script for MADPL is shown in Algorithm 1.

\section{Experimental Setting}

\subsection{Dataset}

MultiWOZ (Budzianowski et al., 2018) is a multidomain, multi-intent task-oriented dialog corpus that contains 7 domains, 13 intents, 25 slot types, 10,483 dialog sessions, and 71,544 dialog turns. During the data collection process, a user is asked to follow a pre-specified user goal, and is allowed to change the goal during the session if necessary, so the collected dialogs are much closer to realworld conversations. The corpus also provides the domain knowledge that defines all the entities and attributes as the external database.

\subsection{Metrics}

Evaluation of a task-oriented dialog system mainly consists of the cost and task success. We count the number of dialog turns to reflect the dialog cost. A user utterance and a subsequent system utterance are regarded as one dialog turn. We utilize two other metrics: inform $F 1$ and match rate to estimate the task success. Both metrics are calculated at the dialog act level. Inform F1 evaluates whether all the requested information has been informed, and match rate checks whether the booked entities match all the indicated constraints given by the user. The overall task success is reached if and only if both inform recall and match rate are 1 .

\subsection{Baselines}

We compare MADPL with a series of baselines that involve both system policy learning and user policy learning. Note that we do not consider any other approaches that use a user simulator for policy training because our motivation is to avoid explicitly modeling a simulator.

SL Supervised Imitation Learning directly uses the dialog act annotations and trains the agents simply by behavior cloning using Eq. 1, which is the same as the pretraining phase in MADPL.

The following three baselines are all RL algorithms that start from the pretrained policy:

RL Independent Reinforcement Learning learns only one dialog policy by fixing another agent following the single RL setting, and the reward for

\begin{tabular}{cccc}
\hline Class & Attraction & Hospital & Hotel \\
Count & 320 & 22 & 389 \\
\hline Police & Restaurant & Taxi & Train \\
22 & 457 & 164 & 421 \\
\hline Num. & Single & Two & Three \\
Count & 328 & 549 & 123 \\
\hline
\end{tabular}

Table 1: Domain distribution of user goals used in the automatic evaluation. A user goal with multiple domains is counted repeatedly for each domain.

the agent is the sum of role-specific reward and global reward. For example, the user policy uses the reward $r=r^{U}+r^{G}$ at each dialog turn.

CRL Centralized Reinforcement Learning is a MARL approach that uses a single centralized critic on the sum of reward $r=r^{U}+r^{S}+r^{G}$ to train two agents simultaneously, which also serves for the ablation test of MADPL.

IterDPL Iterative Dialog Policy Learning (Liu and Lane, 2017) updates two agents iteratively using single RL training to reduce the risk of nonstationarity when jointly training the two agents.

\section{Automatic Evaluation}

\subsection{Interaction between Two Agents}

A set of 1,000 user goals are used for automatic evaluation as shown in Table 1. When the dialog is launched, two agents interact with each other around a given user goal. The performance of interaction between the two trained policies are shown in Table 2. MADPL reaches the highest match rate and task success among all the methods. It manages to improve the success rate of the pretrained policies from $49.7 \%$ to $70.1 \%$. Single RL policies (row 2 to 4 ) have limited improvement, and even decline in match rate since they assume a stationary environment. The comparison between CRL and IterDPL indicates the effectiveness of CTDE in the multi-agent task. The superiority of MADPL against CRL shows that two agents benefit from the role-aware reward decomposition in HVN. The learning curves in Fig. 3 illustrates that the success rate grows rapidly in MADPL, and it always improves the success rate as the training proceeds.

The average reward of each component reward is shown in 4. We run 10 different instances of MADPL with different random seeds. The solid curves correspond to the mean and the shaded region to the standard deviation of rewards over the 


\begin{tabular}{|c|c|c|c|c|c|}
\hline System & User & Turns & Inform & Match & Success \\
\hline SL & SL & 6.34 & 73.08 & 82.58 & 49.7 \\
\hline SL & RL & 8.75 & 76.86 & 76.28 & 60.2 \\
\hline RL & SL & 6.20 & 72.84 & 79.15 & 51.1 \\
\hline RL & RL & 7.92 & 75.96 & 70.37 & 58.7 \\
\hline \multicolumn{2}{|c|}{ CRL } & 8.13 & 68.29 & 89.71 & 66.6 \\
\hline \multicolumn{2}{|c|}{ IterDPL } & 8.79 & 74.01 & 81.04 & 64.6 \\
\hline \multicolumn{2}{|c|}{ MADPL } & 8.96 & 76.26 & 90.98 & 70.1 \\
\hline
\end{tabular}

Table 2: Performance of the interaction between the user agent and the system agent.

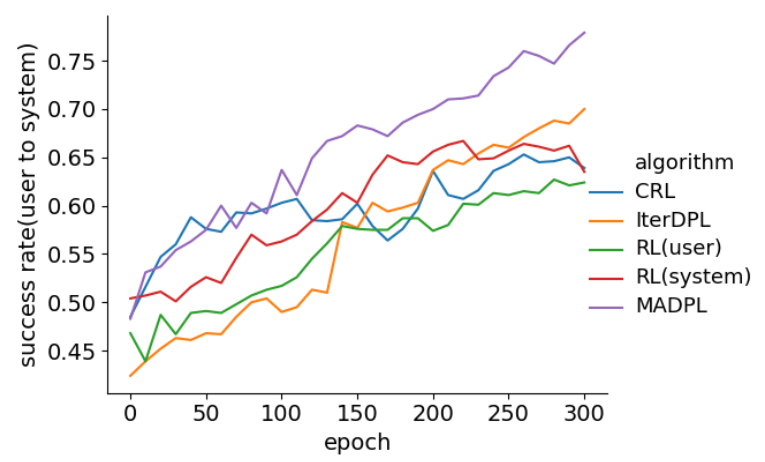

Figure 3: Learning curves of the interaction between the user agent and the system agent.

10 trials. We can observe that all the rewards increase steadily during the training process, which implies that HVN has estimated a proper return for policy training.

\subsection{Interaction with Benchmark Policies}

It is essential to evaluate a multi-agent dialog system whether all the agents understand the semantic interaction rather than invent an uninterpretable language (Kottur et al., 2017; Lee et al., 2019a). To this end, we use two benchmark policies in the standardized task-oriented dialog system platform Convlab (Lee et al., 2019b) to examine all the methods. Each benchmark is a strong rule-based system policy or user policy at the dialog act level, which is used as the simulated evaluation in the DSTC-8 Track 1 competition and show a high correlation with real user interaction (Li et al., 2020). The trained system/user policy in each method is directly deployed to interact with the benchmark user/system policy during the test without any other finetuning, which can be regarded as a weakly zeroshot experiment. The same goal set in Table 1 is used here.

Table 3 and Fig. 5 show the results of the interac-
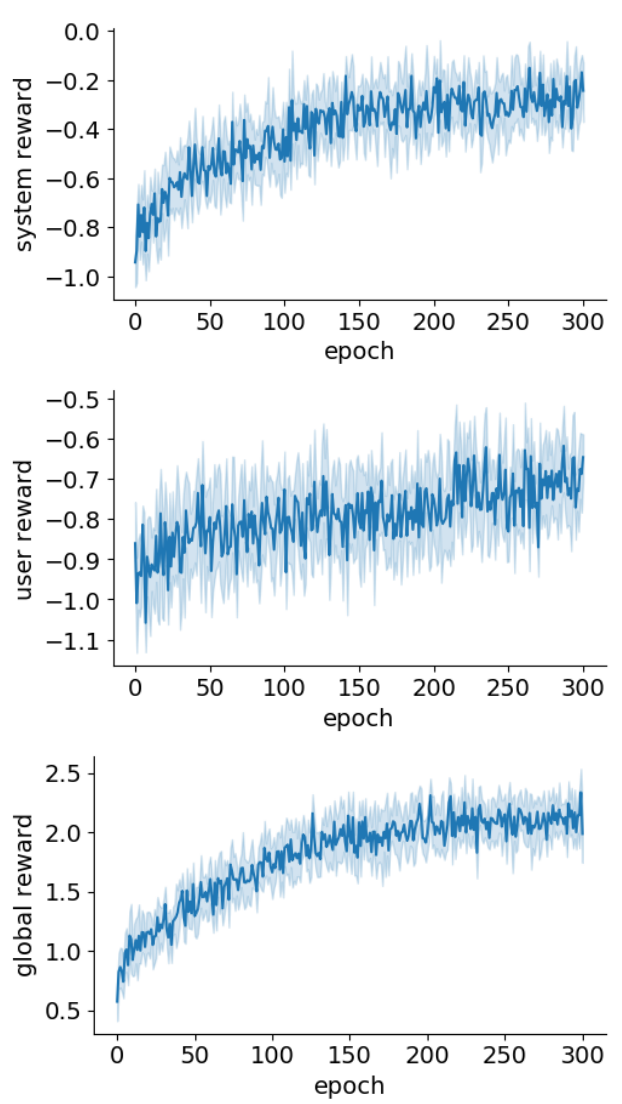

Figure 4: Learning curves of MADPL on system reward (top), user reward (middle) and global reward (bottom).

tion between the benchmark user policy and the system agent of each model. The SOTA performance from GDPL (Takanobu et al., 2019) that directly trains with benchmark user policy is also presented as the soft performance upper bound. Among all the methods, MADPL has achieved the highest task success and the second-highest match rate. All the methods experience a decline in inform $\mathrm{F} 1$ after the RL training. Fig. 5 also shows that the success rate is unstable during training. This is because the action space of the system policy is much larger, thus more challenging to learn. In spite of that, the success rate of MADPL shows a rising trend.

Table 4 and Fig. 6 show the results of the interaction between the user agent of each method and the benchmark system policy. Among all the methods, MADPL has achieved the highest inform F1 and task success. Though CRL improves the performance at the beginning, the success rate fails to increase further afterwards, while MADPL continues to improve all the time. This also indirectly indicates the advantage of using role-aware reward decomposition in HVN. 


\begin{tabular}{c|cccc}
\hline System & Turns & Inform & Match & Success \\
\hline SL & 7.76 & $\mathbf{8 3 . 3 3}$ & 85.84 & 84.2 \\
RL & 7.53 & 82.06 & 85.77 & 84.3 \\
CRL & 8.38 & 72.43 & $\mathbf{8 9 . 4 8}$ & 86.4 \\
IterDPL & 7.74 & 79.68 & 82.49 & 82.5 \\
\hline MADPL & 7.63 & 79.93 & 89.24 & $\mathbf{8 7 . 7}$ \\
GDPL & 7.62 & 92.10 & 91.50 & 92.1 \\
\hline
\end{tabular}

Table 3: Performance of the interaction between the benchmark user policy and each system agent.

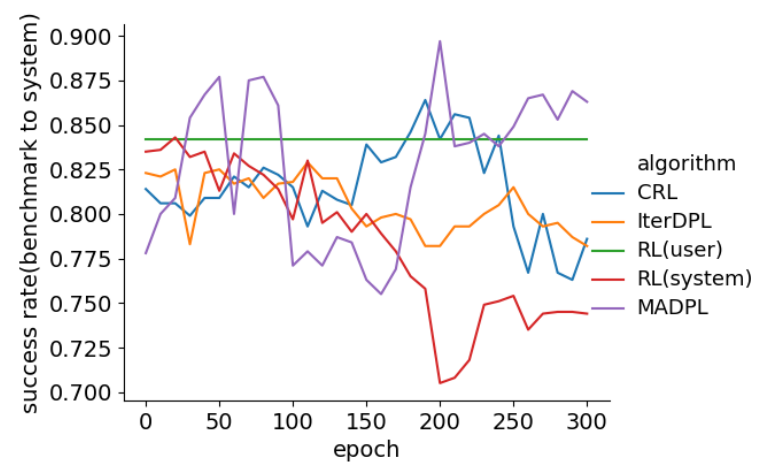

Figure 5: Learning curves of the interaction between the benchmark user policy and each system agent.

\begin{tabular}{c|cccc}
\hline User & Turns & Inform & Match & Success \\
\hline SL & 8.64 & 78.64 & 87.84 & 51.7 \\
RL & 11.18 & 85.69 & 92.13 & 77.2 \\
CRL & 11.31 & 86.58 & $\mathbf{9 2 . 8 9}$ & 74.7 \\
IterDPL & 12.53 & 84.68 & 92.57 & 75.5 \\
\hline MADPL & 13.25 & $\mathbf{8 7 . 0 4}$ & 90.81 & $\mathbf{8 3 . 7}$ \\
\hline
\end{tabular}

Table 4: Performance of the interaction between each user agent and the benchmark system policy.

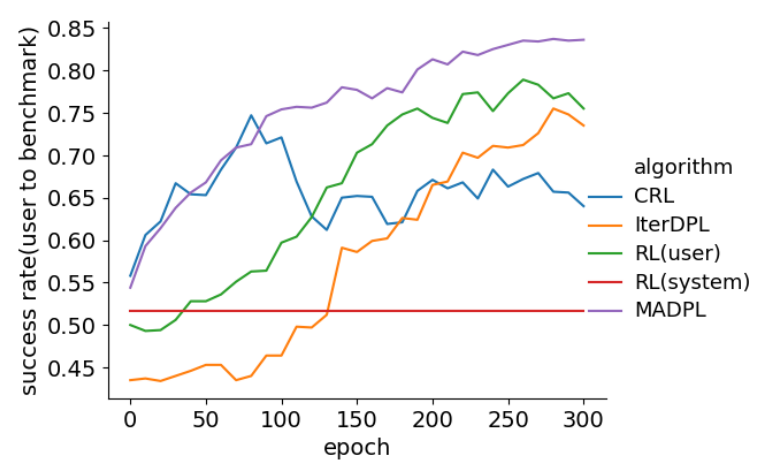

Figure 6: Learning curves of the interaction between each user agent and the benchmark system policy.

In summary, each policy trained from MADPL can interact well with the benchmark policy, which

\begin{tabular}{|c|c|c|c|c|c|c|c|c|c|}
\hline \multirow{2}{*}{ VS. } & \multicolumn{3}{|c|}{ System Q } & \multicolumn{3}{|c|}{ User Q } & \multicolumn{3}{|c|}{ Success } \\
\hline & W & D & $\mathrm{L}$ & W & D & $\mathrm{L}$ & W & $\mathrm{D}$ & $\mathrm{L}$ \\
\hline SL/SL & 55 & 22 & 23 & 61 & 25 & 14 & 68 & 26 & 6 \\
\hline RL/RL & 49 & 23 & 28 & 52 & 28 & 20 & 70 & 19 & 11 \\
\hline IterDPL & 50 & 27 & 23 & 56 & 30 & 14 & 64 & 24 & 12 \\
\hline
\end{tabular}

Table 5: Human preference on dialog session pairs that MADPL wins (W), draws with (D) or loses to (L) baselines with regard to quality (Q) and success by majority voting.

implies that MADPL learns a reasonable dialog strategy.

\subsection{Goal across Multiple Domains}

We also investigate the domains in the user goals to observe the scalability of each method in the complex tasks. 200 goals are randomly sampled under each setting. Fig. 7 presents the results of the interaction between two agents in different numbers or classes of domains. The success rate decreases substantially as the number of domains increases in the goal. When there are 3 domains in the goal, RL/RL gets a high inform F1 but a low match rate, IterDPL gets a high match rate but a low inform F1, while MADPL can still keep a high inform F1 and match rate, and obtains the highest task success. In terms of the class of domains, there are 7/10/6 informable slots that needs to be tracked in the Restaurant/Hotel/Train domain respectively. Among these, MADPL outperforms other baselines in the Restaurant and Hotel domains, and performs comparably in the Train domain. In brief, all the results indicate that MADPL has good scalability in multi-domain dialog.

\section{Human Evaluation}

For human evaluation, we hire Amazon Mechanical Turkers to conduct pairwise comparison between MADPL and baselines. Since all the policies work at the dialog act level, we generate the texts from dialog acts using hand-crafted templates to make the dialog readable. Each Turker is asked to read a user goal first, then we show 2 dialog sessions around this user goal, one from MADPL and the other from another baseline. We randomly sample 100 goals for each baseline. For each goal, 5 Turkers are asked to judge which dialog is better (win, draw or lose) according to different subjective assessments independently: (I) system quality, (II) user quality, 

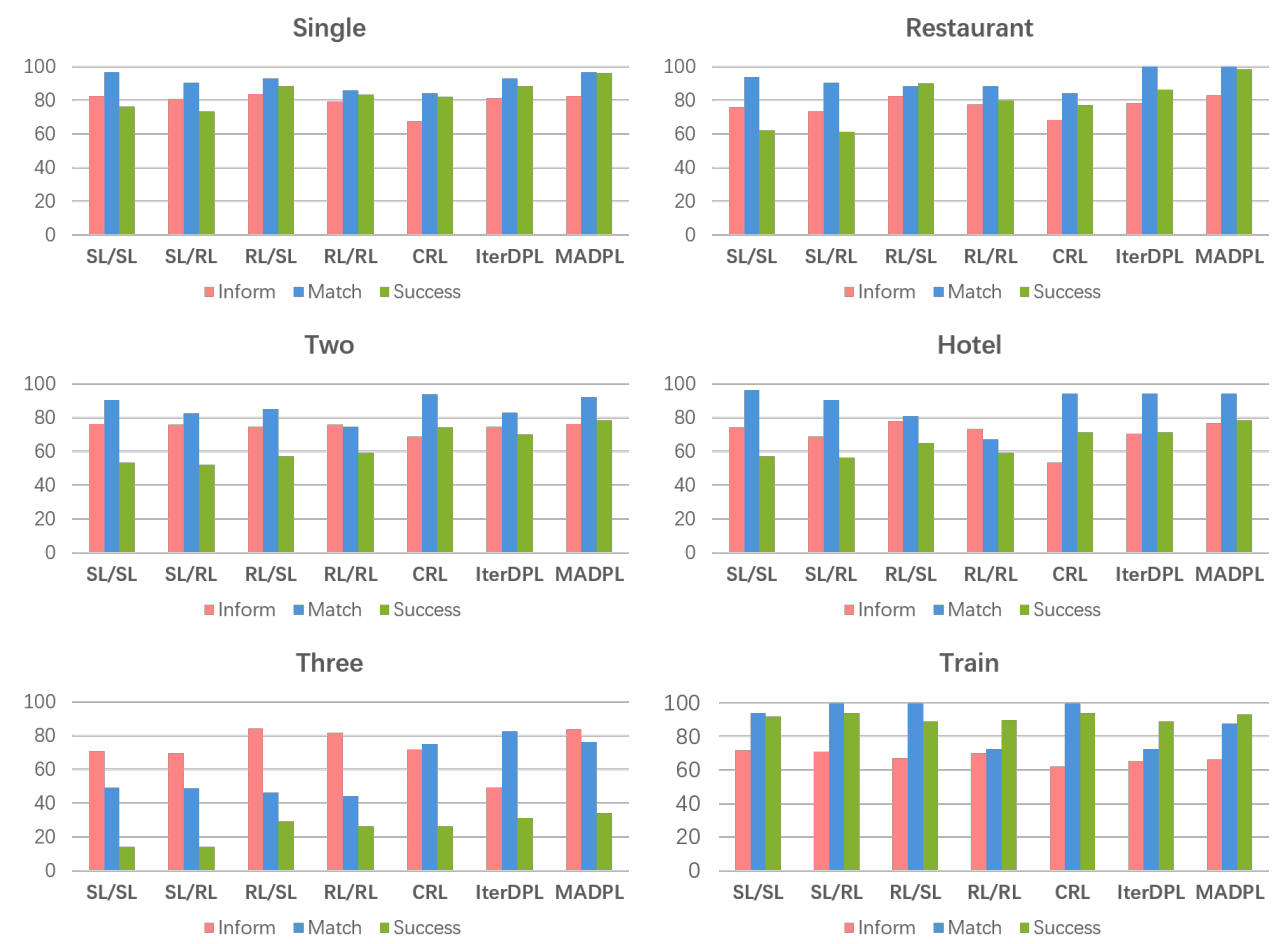

Figure 7: Performance of dialog agents according to the different number (left) or class (right) of domains in the dialog.

and (III) task success. The system quality metric evaluates whether the system policy provides the user with the required information efficiently, and the user quality metric evaluates whether the user policy expresses the constraints completely in an organized way. Note that we do not evaluate the quality of language generation here.

Table 5 shows the results of human preference by majority voting. We can observe that the high win rate of MADPL on the task success is consistent with the results of automatic evaluation, and MADPL outperforms three baselines significantly in all aspects (sign test, p-value $<0.01$ ) except for the system quality against RL/RL policies.

The proportion of the pairwise annotations in which at least 3 of 5 annotators assign the same label to a task is $78.7 \% / 77.3 \% / 83.3 \%$ for system quality/user quality/task success, respectively. This indicates that annotators have moderate agreements. The human judgements align well with the results of automatic evaluation, which also indicates the reliability of the metrics used in task-oriented dia$\log$.

\section{Conclusion}

We present a multi-agent dialog policy algorithm, MADPL, that trains the user policy and the sys- tem policy simultaneously. It uses the actor-critic framework to facilitate pretraining and bootstrap RL training in multi-domain task-oriented dialog. We also introduce role-aware reward decomposition to integrate the task knowledge into the algorithm. MADPL enables the developers to set up a dialog system rapidly from scratch. It only requires the annotation of dialog acts in the corpus for pretraining and does not need to build a user simulator explicitly beforehand. Extensive experiments ${ }^{1}$ demonstrate the effectiveness, reasonableness and scalability of MADPL.

As future work, we will apply MADPL in the more complex dialogs and verify the role-aware reward decomposition in other dialog scenarios.

\section{Acknowledgement}

This work was jointly supported by the NSFC projects (Key project with No. 61936010 and regular project with No. 61876096), and the National Key R\&D Program of China (Grant No. 2018YFC0830200). We would like to thank THUNUS NExT Joint-Lab for the support. The code is available at https://github.com/ truthless11/MADPL.

\footnotetext{
${ }^{1}$ We provide implementation details and case studies in appendix.
} 


\section{References}

Andrew G Barto, Richard S Sutton, and Charles W Anderson. 1983. Neuronlike adaptive elements that can solve difficult learning control problems. IEEE transactions on systems, man, and cybernetics, 13(5):834-846.

Daniel S Bernstein, Robert Givan, Neil Immerman, and Shlomo Zilberstein. 2002. The complexity of decentralized control of markov decision processes. Mathematics of operations research, 27(4):819-840.

Paweł Budzianowski, Tsung-Hsien Wen, Bo-Hsiang Tseng, Iñigo Casanueva, Stefan Ultes, Osman Ramadan, and Milica Gašić. 2018. Multiwoz: A largescale multi-domain wizard-of-oz dataset for taskoriented dialogue modelling. In 2018 Conference on Empirical Methods in Natural Language Processing, pages 5016-5026.

Abhishek Das, Satwik Kottur, José MF Moura, Stefan Lee, and Dhruv Batra. 2017. Learning cooperative visual dialog agents with deep reinforcement learning. In 2017 IEEE International Conference on Computer Vision, pages 2951-2960.

Layla El Asri, Jing He, and Kaheer Suleman. 2016. A sequence-to-sequence model for user simulation in spoken dialogue systems. 17th Annual Conference of the International Speech Communication Association, pages 1151-1155.

Mehdi Fatemi, Layla El Asri, Hannes Schulz, Jing He, and Kaheer Suleman. 2016. Policy networks with two-stage training for dialogue systems. In 17th Annual Meeting of the Special Interest Group on Discourse and Dialogue, pages 101-110.

Jun Feng, Heng Li, Minlie Huang, Shichen Liu, Wenwu Ou, Zhirong Wang, and Xiaoyan Zhu. 2018. Learning to collaborate: Multi-scenario ranking via multi-agent reinforcement learning. In 27th International Conference on World Wide Web, pages 19391948.

Jakob N Foerster, Gregory Farquhar, Triantafyllos Afouras, Nantas Nardelli, and Shimon Whiteson. 2018. Counterfactual multi-agent policy gradients. In 32nd AAAI Conference on Artificial Intelligence, pages 2974-2982.

Kallirroi Georgila, Claire Nelson, and David Traum. 2014. Single-agent vs. multi-agent techniques for concurrent reinforcement learning of negotiation dialogue policies. In 52nd Annual Meeting of the Association for Computational Linguistics, pages 500 510.

Izzeddin Gür, Dilek Hakkani-Tür, Gokhan Tür, and Pararth Shah. 2018. User modeling for task oriented dialogues. In 2018 IEEE Spoken Language Technology Workshop, pages 900-906.

He He, Derek Chen, Anusha Balakrishnan, and Percy Liang. 2018. Decoupling strategy and generation in negotiation dialogues. In 2018 Conference on Empirical Methods in Natural Language Processing, pages 2333-2343.

Simon Keizer, Milica Gašić, Filip Jurčíček, François Mairesse, Blaise Thomson, Kai Yu, and Steve Young. 2010. Parameter estimation for agendabased user simulation. In 11th Annual Meeting of the Special Interest Group on Discourse and Dialogue, pages 116-123.

Satwik Kottur, José Moura, Stefan Lee, and Dhruv Batra. 2017. Natural language does not emerge naturally in multi-agent dialog. In 2017 Conference on Empirical Methods in Natural Language Processing, pages 2962-2967.

Florian Kreyssig, Iñigo Casanueva, Paweł Budzianowski, and Milica Gasic. 2018. Neural user simulation for corpus-based policy optimisation of spoken dialogue systems. In 19th Annual Meeting of the Special Interest Group on Discourse and Dialogue, pages 60-69.

Jason Lee, Kyunghyun Cho, and Douwe Kiela. 2019a. Countering language drift via visual grounding. In 2019 Conference on Empirical Methods in Natural Language Processing and 9th International Joint Conference on Natural Language Processing, pages 4376-4386.

Sungjin Lee, Qi Zhu, Ryuichi Takanobu, Zheng Zhang, Yaoqin Zhang, Xiang Li, Jinchao Li, Baolin Peng, Xiujun Li, Minlie Huang, and Jianfeng Gao. 2019b. Convlab: Multi-domain end-to-end dialog system platform. In 57th Annual Meeting of the Association for Computational Linguistics: System Demonstrations, pages 64-69.

Mike Lewis, Denis Yarats, Yann Dauphin, Devi Parikh, and Dhruv Batra. 2017. Deal or no deal? end-toend learning of negotiation dialogues. In 2017 Conference on Empirical Methods in Natural Language Processing, pages 2443-2453.

Jinchao Li, Baolin Peng, Sungjin Lee, Jianfeng Gao, Ryuichi Takanobu, Qi Zhu, Minlie Huang, Hannes Schulz, Adam Atkinson, and Mahmoud Adada. 2020. Results of the multi-domain task-completion dialog challenge. In 34th AAAI Conference on Artificial Intelligence, Eighth Dialog System Technology Challenge Workshop.

Bing Liu and Ian Lane. 2017. Iterative policy learning in end-to-end trainable task-oriented neural dialog models. In 2017 IEEE Automatic Speech Recognition and Understanding Workshop, pages 482-489.

Ryan Lowe, Yi Wu, Aviv Tamar, Jean Harb, Pieter Abbeel, and Igor Mordatch. 2017. Multi-agent actor-critic for mixed cooperative-competitive environments. In 31st Annual Conference on Neural Information Processing Systems, pages 6379-6390. 
Volodymyr Mnih, Koray Kavukcuoglu, David Silver, Andrei A Rusu, Joel Veness, Marc G Bellemare, Alex Graves, Martin Riedmiller, Andreas K Fidjeland, Georg Ostrovski, Stig Petersen, Charles Beattie, Amir Sadik, Ioannis Antonoglou, Helen King, Dharshan Kumaran, Daan Wierstra, Shane Legg, and Demis Hassabis. 2015. Human-level control through deep reinforcement learning. Nature, 518(7540):529-533.

Alexandros Papangelis, Yi-Chia Wang, Piero Molino, and Gokhan Tur. 2019. Collaborative multi-agent dialogue model training via reinforcement learning. In 20th Annual Meeting of the Special Interest Group on Discourse and Dialogue, pages 92-102.

Baolin Peng, Xiujun Li, Lihong Li, Jianfeng Gao, Asli Celikyilmaz, Sungjin Lee, and Kam-Fai Wong. 2017. Composite task-completion dialogue policy learning via hierarchical deep reinforcement learning. In 2017 Conference on Empirical Methods in Natural Language Processing, pages 2231-2240.

Tabish Rashid, Mikayel Samvelyan, Christian Schroeder Witt, Gregory Farquhar, Jakob Foerster, and Shimon Whiteson. 2018. Qmix: Monotonic value function factorisation for deep multi-agent reinforcement learning. In 35th International Conference on Machine Learning, pages 4292-4301.

Jost Schatzmann, Blaise Thomson, Karl Weilhammer, Hui Ye, and Steve Young. 2007. Agenda-based user simulation for bootstrapping a pomdp dialogue system. In 2007 Conference of the North American Chapter of the Association for Computational Linguistics: Human Language Technologies, pages 149-152.

Pararth Shah, Dilek Hakkani-Tür, Bing Liu, and Gokhan Tür. 2018. Bootstrapping a neural conversational agent with dialogue self-play, crowdsourcing and on-line reinforcement learning. In 2018 Conference of the North American Chapter of the Association for Computational Linguistics: Human Language Technologies, pages 41-51.

Weiyan Shi, Kun Qian, Xuewei Wang, and Zhou Yu. 2019. How to build user simulators to train rl-based dialog systems. In 2019 Conference on Empirical Methods in Natural Language Processing and 9th International Joint Conference on Natural Language Processing, pages 1990-2000.

David Silver, Julian Schrittwieser, Karen Simonyan, Ioannis Antonoglou, Aja Huang, Arthur Guez, Thomas Hubert, Lucas Baker, Matthew Lai, Adrian Bolton, Yutian Chen, Timothy Lillicrap, Fan Hui, Laurent Sifre, George Van Den Driessche, Thore Graepel, and Demis Hassabis. 2017. Mastering the game of go without human knowledge. Nature, 550(7676):354-359.

Andreas Stolcke, Klaus Ries, Noah Coccaro, Elizabeth Shriberg, Rebecca Bates, Daniel Jurafsky, Paul
Taylor, Rachel Martin, Carol Van Ess-Dykema, and Marie Meteer. 2000. Dialogue act modeling for automatic tagging and recognition of conversational speech. Computational linguistics, 26(3):339-373.

Pei-Hao Su, Milica Gašić, Nikola Mrkšić, Lina M Rojas Barahona, Stefan Ultes, David Vandyke, TsungHsien Wen, and Steve Young. 2016. On-line active reward learning for policy optimisation in spoken dialogue systems. In 54th Annual Meeting of the Association for Computational Linguistics, pages 24312441.

Shang-Yu Su, Xiujun Li, Jianfeng Gao, Jingjing Liu, and Yun-Nung Chen. 2018. Discriminative deep dyna-q: Robust planning for dialogue policy learning. In 2018 Conference on Empirical Methods in Natural Language Processing, pages 3813-3823.

Ryuichi Takanobu, Hanlin Zhu, and Minlie Huang. 2019. Guided dialog policy learning: Reward estimation for multi-domain task-oriented dialog. In 2019 Conference on Empirical Methods in Natural Language Processing and 9th International Joint Conference on Natural Language Processing, pages 100-110.

Harm Van Seijen, Mehdi Fatemi, Joshua Romoff, Romain Laroche, Tavian Barnes, and Jeffrey Tsang. 2017. Hybrid reward architecture for reinforcement learning. In 31st Annual Conference on Neural Information Processing Systems, pages 5392-5402.

Jason D Williams, Antoine Raux, and Matthew Henderson. 2016. The dialog state tracking challenge series: A review. Dialogue \& Discourse, 7(3):4-33.

Zhirui Zhang, Xiujun Li, Jianfeng Gao, and Enhong Chen. 2019. Budgeted policy learning for taskoriented dialogue systems. In 57th Annual Meeting of the Association for Computational Linguistics, pages 3742-3751.

Tiancheng Zhao, Kaige Xie, and Maxine Eskenazi. 2019. Rethinking action spaces for reinforcement learning in end-to-end dialog agents with latent variable models. In 2019 Conference of the North American Chapter of the Association for Computational Linguistics: Human Language Technologies, pages 1208-1218. 


\section{A Implementation Details}

Both the system policy $\pi$ and the user policy $\mu$ are implemented with two hidden layer MLPs. The action space of system policy and user policy is 172 and 80 respectively. For Hybrid Value Network $V$, all neural network units $f(\cdot)$ are two hidden layer MLPs. The activation function is all Relu for MLPs.

We use RMSprop as the optimization algorithm. The batch size is set to 32 . The weighted pretraining factor $\beta$ is $2.5,4$ for the system policy and user policy respectively. The learning rate for two polices is 1e-3 when pretraining. As for RL training, the learning rate is $1 e-4,5 e-5$ for the system policy and the user policy respectively, and 3e-5 for Hybrid Value Network. The discount factor $\gamma$ is 0.99 , and the target network is updated every $C=$ 400 training iterations.

In terms of reward design, the empty action penalty is set to -5 , and penalties of other types are set to -1 . The sub-goal completion reward is set to 5. The task success and the user goal reward are set to 20 if triggered, otherwise they are set to -5 .

\section{B Case Study}

To illustrate the superiority of our model, we show a pair of sample dialog sessions between the user and the system, trained with RL/RL and MADPL, respectively. As shown in Table 6, the user goal contains two domains (i.e., train and restaurant). The user agent is supposed to inform the constraints displayed in Info, request information displayed in Reqt, and express the intent to book the train and restaurant with the information in Book. The system agent is required to answer the request, as well as book the train and the restaurant according to the user's demands. Table 7 shows the sampled dia$\log$ sessions. For RL/RL, the user sometimes talks about both the train and the restaurant simultaneously, making it difficult for the system to respond (highlighted in red color). Moreover, the system agent in RL/RL sometimes fails to answer the requested information or book the restaurant as the user demands (highlighted in blue color). As a result, the dialog in RL/RL fails as the user does not request the arrival time of the train, and the system does not book the restaurant for the user. In comparison, both the user and the system in MADPL fulfill their own task, and the whole dialog is successful. Table 8 shows the same sample dialog sessions with the original dialog acts. 


\begin{tabular}{c|c|c|c|c|c}
\hline \multicolumn{2}{c|}{ Train } & \multicolumn{3}{c}{ Restaurant } \\
\hline Info & Reqt & Book & Info & Reqt & Book \\
\hline $\begin{array}{c}\text { destination=peterborough } \\
\text { day=thursday } \\
\begin{array}{c}\text { departure=cambridge } \\
\text { leaveAt=19:45 }\end{array}\end{array}$ & $\begin{array}{c}\text { duration } \\
\text { arriveBy }\end{array}$ & people=3 & $\begin{array}{c}\text { food=european } \\
\text { pricerange=expensive }\end{array}$ & $\begin{array}{c}\text { phone } \\
\text { postcode } \\
\text { address }\end{array}$ & $\begin{array}{c}\text { time=19:30 } \\
\text { people=5 } \\
\text { day=tuesday }\end{array}$ \\
\hline
\end{tabular}

Table 6: User goal used in the case study.

\begin{tabular}{|c|c|}
\hline RL/RL & MADPL \\
\hline $\begin{array}{l}\text { U: Hi, I'd like to take a train from cambridge to peterbor- } \\
\text { ough on thursday. I want to leave at about 19:45. }\end{array}$ & $\begin{array}{l}\text { U: Hi, I'd like to take a train from cambridge to peterbor- } \\
\text { ough on thursday. I want to leave at about 19:45. }\end{array}$ \\
\hline $\begin{array}{l}\text { S: You may consider TR7324, which leaves at } 21: 40 \text {. Shall } \\
\text { I book tickets for you? }\end{array}$ & $\begin{array}{l}\text { S: You may consider TR7324, which leaves at } 21: 40 \text {, and } \\
\text { arrives at cambridge at } 24: 23 \text {. Shall I book tickets for } \\
\text { you? }\end{array}$ \\
\hline $\begin{array}{l}\text { U: Sounds good! Please book tickets to peterborough for } 3 \\
\text { people. }\end{array}$ & U: Sounds good! Please book tickets for 3 people. \\
\hline $\begin{array}{l}\text { S: I've booked your train tickets. The price is } 10.10 \text { pounds } \\
\text { and your reference number is train-00002321. Is there } \\
\text { anything else I can help you with today? }\end{array}$ & $\begin{array}{l}\text { S: I've booked your train tickets. The price is } 10.10 \text { pounds } \\
\text { and your reference number is train-00002321. Is there } \\
\text { anything else I can help you with today? }\end{array}$ \\
\hline $\begin{array}{l}\text { U: Could you please tell me the train ID and travel time? I } \\
\text { also want to book an european restaurant with expensive } \\
\text { price. }\end{array}$ & $\begin{array}{l}\text { U: I also want to book an european restaurant with expen- } \\
\text { sive price on Tuesday. }\end{array}$ \\
\hline $\begin{array}{l}\text { S: There are } 4 \text { choices for expensive european restaurant. } \\
\text { What area of town would you prefer? }\end{array}$ & $\begin{array}{l}\text { S: There are } 4 \text { choices found. City Stop Restaurant is a } \\
\text { good choice. }\end{array}$ \\
\hline $\begin{array}{l}\text { U: I don't care about the location. Could you please tell me } \\
\text { the address, phone number and post of the restaurant? }\end{array}$ & $\begin{array}{l}\text { U: I don't care its name, but make sure it's an expensive } \\
\text { restaurant. Can you help me with a reservation for } 5 \\
\text { people at 19:30 this coming Tuesday? }\end{array}$ \\
\hline $\begin{array}{l}\text { S: Sure, City Stop Restaurant is located at Cambridge City } \\
\text { Football Club Milton Road Chesterton and the post is } \\
\text { cb4luy. The phone number is } 01223363270 . \text { Is there } \\
\text { anything else I can help you with today? }\end{array}$ & $\begin{array}{l}\text { S: Booking was successful. Reference number is: } \\
\text { restaurant- } 00000083 \text {. }\end{array}$ \\
\hline $\begin{array}{l}\text { U: Can you help me with a reservation for } 5 \text { people at } 19: 30 \\
\text { this coming Tuesday? }\end{array}$ & $\begin{array}{l}\text { U: Although I don't care its name, I'd like to know its phone } \\
\text { number and post. }\end{array}$ \\
\hline S: Is there anything else I can help you with? & $\begin{array}{l}\text { S: Sure, the phone number is } 01223368786 \text { and the post is } \\
\text { cb23rh. Anything else? }\end{array}$ \\
\hline $\begin{array}{l}\text { U: Could you please tell me the travel time of the train I } \\
\text { just booked? }\end{array}$ & U: What's the address of the restaurant? \\
\hline $\begin{array}{l}\text { S: The travel time is } 60 \text { minutes. Anything else I can look } \\
\text { up for you? }\end{array}$ & $\begin{array}{l}\text { S: The restaurant is located at Cambridge Lodge Hotel } 139 \\
\text { Huntingdon Road City Centre. Is there anything else I } \\
\text { can help you with? }\end{array}$ \\
\hline U: Thanks! When will the train arrive? & $\begin{array}{l}\text { U: Could you please tell me the travel time of the train I } \\
\text { just booked? Thanks very much! }\end{array}$ \\
\hline S: You're welcome! Anything else? Bye! & $\begin{array}{l}\text { S: The travel time is } 105 \text { minutes. Anything else I can look } \\
\text { up for you? }\end{array}$ \\
\hline U: Thank you very much! & U: Thank you. That's all I needed. \\
\hline S: You are very welcome. Goodbye. & S: You are very welcome. Goodbye. \\
\hline $\begin{array}{l}\text { Failure: User does not request the arrive time of the train, and } \\
\text { the system does not book the restaurant for the user. }\end{array}$ & Success \\
\hline
\end{tabular}

Table 7: A sample dialog session comparison between RL/RL and MADPL in natural language. 


\begin{tabular}{|c|c|}
\hline RL/RL & MADPL \\
\hline $\begin{array}{l}\text { U: \{'train-inform-dest': 'peterborough', 'train-inform- } \\
\text { day': 'thursday', 'train-inform-depart': 'cambridge', } \\
\text { 'train-inform-leave': '19:45'\} }\end{array}$ & $\begin{array}{l}\text { U: }\{\text { 'train-inform-dest': 'peterborough', 'train-inform- } \\
\text { day': 'thursday', 'train-inform-depart': 'cambridge', } \\
\text { 'train-inform-leave': '19:45'\} }\end{array}$ \\
\hline $\begin{array}{l}\text { S: \{'train-inform-leave': '21:40', 'train-inform-id': } \\
\text { 'TR7324', 'train-offerbook-none': 'none' }\}\end{array}$ & $\begin{array}{l}\text { S: }\{\text { 'train-inform-leave': '21:40', 'train-inform-id': } \\
\text { 'TR7324', 'train-inform-arrive': '24:23', 'train- } \\
\text { offerbook-none': 'none', 'train-inform-dest': 'cam- } \\
\text { bridge'\} }\end{array}$ \\
\hline $\begin{array}{l}\text { U: \{'train-inform-dest': 'peterborough', 'train-inform- } \\
\text { people': '3'\} }\end{array}$ & U: $\{$ 'train-inform-people': '3'\} \\
\hline $\begin{array}{rlr}\text { S: } & \{\text { 'general-reqmore-none': 'none', } & \text { 'train- } \\
& \text { offerbooked-ref': 'train-00002321', } & \text { 'train- } \\
& \text { offerbooked-ticket': '10.10 pounds'\} } & \end{array}$ & $\begin{array}{rlr}\text { S: } & \{\text { 'general-reqmore-none': 'none', } & \text { 'train- } \\
& \text { offerbooked-ref': 'train-00002321', } & \text { 'train- } \\
& \text { offerbooked-ticket': '10.10 pounds'\} } & \end{array}$ \\
\hline $\begin{array}{l}\text { U: \{'restaurant-inform-food': 'european', 'restaurant- } \\
\text { inform-price': 'expensive', 'train-request-time': '?', } \\
\text { 'train-request-id': '?'\} }\end{array}$ & $\begin{array}{l}\text { U: \{'restaurant-inform-food': 'european', 'restaurant- } \\
\text { inform-price': 'expensive', 'restaurant-inform-day': } \\
\text { 'tuesday'\} }\end{array}$ \\
\hline $\begin{array}{l}\text { S: \{'restaurant-inform-choice': '4', 'restaurant-inform- } \\
\text { food': 'european', 'restaurant-inform-price': 'expen- } \\
\text { sive', 'restaurant-request-area-?': '?'\} }\end{array}$ & 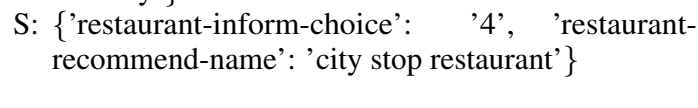 \\
\hline $\begin{array}{l}\text { U: \{'restaurant-inform-area': 'dont care', 'restaurant- } \\
\text { request-addr': '?', 'restaurant-request-phone': '?', } \\
\text { 'restaurant-request-post': '?'\} }\end{array}$ & $\begin{array}{l}\text { U: \{'restaurant-inform-price': 'expensive', 'restaurant- } \\
\text { inform-time': '19:30', 'restaurant-inform-day': } \\
\text { 'tuesday', 'restaurant-inform-people': } \\
\text { 'restaurant-inform-name': 'dont care'\} }\end{array}$ \\
\hline $\begin{array}{l}\text { S: \{'general-reqmore-none': 'none', 'restaurant- } \\
\text { inform-name': 'city stop restaurant', 'restaurant- } \\
\text { inform-addr': 'Cambridge City Football Club } \\
\text { Milton Road Chesterton', 'restaurant-recommend- } \\
\text { name': 'city stop restaurant', 'restaurant-inform- } \\
\text { phone': '01223363270', 'restaurant-inform-post': } \\
\text { 'cb4luy'\} }\end{array}$ & $\begin{array}{l}\text { S: \{'general-reqmore-none': 'none', 'booking-book- } \\
\text { ref': 'restaurant-00000083'\} }\end{array}$ \\
\hline $\begin{array}{l}\text { U: \{'restaurant-inform-time': '19:30', 'restaurant- } \\
\text { inform-day': 'tuesday', 'restaurant-inform-people': } \\
\text { '5' }\end{array}$ & $\begin{array}{l}\text { U: \{'restaurant-inform-name': 'dont care', 'restaurant- } \\
\text { request-phone': '?', 'restaurant-request-post': '?'\} }\end{array}$ \\
\hline S: $\{$ 'general-reqmore-none': 'none'\} & $\begin{array}{l}\text { S: \{'general-reqmore-none': 'none', 'restaurant- } \\
\text { inform-phone': '01223368786', 'restaurant-inform- } \\
\text { post': 'cb23rh'\} }\end{array}$ \\
\hline $\mathrm{U}:\{$ 'train-request-time': '?'\} & U: \{'restaurant-request-addr': '?'\} \\
\hline $\begin{array}{l}\text { S: \{'general-reqmore-none': 'none', 'train-inform- } \\
\text { time': '60 minutes'\} }\end{array}$ & $\begin{array}{l}\text { S: \{'general-reqmore-none': 'none', 'restaurant- } \\
\text { inform-addr': 'Cambridge Lodge Hotel } 139 \text { Hunt- } \\
\text { ingdon Road City Centre'\} }\end{array}$ \\
\hline $\begin{array}{l}\text { U: \{'general-thank-none': 'none', 'train-request- } \\
\text { arrive': '?'\} }\end{array}$ & $\begin{array}{l}\text { U: \{'general-thank-none': 'none', 'train-request-time': } \\
\text { ??'\} }\end{array}$ \\
\hline $\begin{array}{l}\text { S: \{'general-reqmore-none': 'none', 'general-bye- } \\
\text { none': 'none', 'general-welcome-none': 'none'\} }\end{array}$ & $\begin{array}{l}\text { S: \{'general-reqmore-none': 'none', 'train-inform- } \\
\text { time': '105 minutes'\} }\end{array}$ \\
\hline $\mathrm{U}:\{$ 'general-thank-none': 'none'\} & U: \{'general-thank-none': 'none'\} \\
\hline $\begin{array}{l}\text { S: \{'general-bye-none': 'none', 'general-welcome- } \\
\text { none': 'none'\} }\end{array}$ & $\begin{array}{l}\text { S: \{'general-bye-none': 'none', 'general-welcome- } \\
\text { none': 'none'\} }\end{array}$ \\
\hline $\begin{array}{l}\text { Failure: User does not request the arrive time of the train, and } \\
\text { the system does not book the restaurant for the user. }\end{array}$ & Success \\
\hline
\end{tabular}

Table 8: A sample dialog session comparison between RL/RL and MADPL in dialog acts. 